\title{
Volume-outcome relationships in coronary artery bypass graft surgery patients: 5-year major cardiovascular event outcomes
}

\author{
Herng-Ching Lin, PhD, ${ }^{a}$ Sudha Xirasagar, PhD, MBBS, ${ }^{b}$ Nai-Wen Tsao, MD, ${ }^{c}$ Yi-Ting Hwang, PhD, ${ }^{d}$ \\ Nai-Wen Kuo, PhD, a and Hsin-Chien Lee, MD, MPH
}

From the School of Health Care Administration, ${ }^{\mathrm{a}}$ Taipei Medical University, Taipei, Taiwan; the Department of Health Services Policy and Management, ${ }^{\mathrm{b}}$ Arnold School of Public Health, University of South Carolina, Columbia, SC; the Division of Cardiovascular Surgery, ${ }^{\mathrm{c}}$ Taipei Medical University Hospital, Taipei, Taiwan; the Department of Statistics, ${ }^{\mathrm{d}}$ National Taipe University, Taipei, Taiwan; and Department of Psychiatry, ${ }^{\mathrm{e}}$ Taipei Medical University and Hospital, Taipei, Taiwan.

This study was supported partially by a grant from the National Science Council (NSC 952416-H-038-001) in Taiwan. This study is based in part on data from the National Health Insurance Research Database provided by the Bureau of National Health Insurance, Department of Health, Taiwan and managed by the National Health Research Institutes. The interpretations and conclusions contained herein do not represent those of the Bureau of National Health Insurance, Department of Health, or the National Health Research Institutes.

Received for publication Aug 6, 2007; accepted for publication Oct 4, 2007.

Address for reprints: Hsin-Chien Lee, MD, Department of Psychiatry, Taipei Medical University and Hospital, 252 Wu-Hsing St., Taipei 110, Taiwan (E-mail: ellalee@ tmu.edu.tw).

J Thorac Cardiovasc Surg 2008;135:923-30 0022-5223/\$34.00

Copyright $\odot 2008$ by The American Association for Thoracic Surgery

doi:10.1016/j.jtcvs.2007.10.005
Objective: Using nationwide population-based data from Taiwan's National Health Insurance database, we examined the association between hospitals' coronary artery bypass grafting surgery volume and 5-year major adverse cardiovascular events.

Methods: We used Taiwan's National Health Insurance claims data linked to the Cause of Death file for the years approximately 1997 to 2004. All 5718 patients who underwent nonemergency coronary artery bypass grafting operations during 1997 through 1999 were classified into one of 4 hospital volume groups: 282 cases or less (low volume, $\mathrm{n}=1584$ patients), 283 to 517 cases (medium volume, $\mathrm{n}=$ 1317), 518 to 725 cases (high volume, $\mathrm{n}=1437$ ), and 726 cases or more (very high volume, $\mathrm{n}=1380$ ).

Results: Increasing hospital volume is associated with increasing 5-year major adverse cardiovascular event-free survival $(72.0 \%, 75.5 \%, 76.9 \%$, and $79.4 \%$ in low-volume, medium-volume, high-volume, and very high-volume hospitals, respectively). Cox regression analysis shows that increasing hospital volume predicts a systematic decrease in adjusted major adverse cardiovascular event hazard at 5 years. The 5-year major adverse cardiovascular event hazard ratios for high-volume and very high-volume hospitals were 0.884 (95\% confidence interval, 0.809-0.965) and 0.811 (95\% confidence interval, 0.728-0.904) relative to low-volume hospitals after adjusting for patient demographics and economic status, initial case severity, coronary artery bypass grafting procedure attributes, and hospital characteristics.

Conclusions: The findings suggest that high-volume hospitals have some processes, infrastructure/personnel factors, or both that seem to produce not only better shortterm outcomes but also better long-term outcomes.

$\mathrm{T}$ There is a growing recognition of the relatively better postoperative outcomes for high-risk procedures with increasing provider volumes. Policymakers internationally have responded with regionalization policies and incentives to concentrate the volumes of high-risk procedures in a few hospitals. In Canada and the United Kingdom, a policy of regionalization of high-risk procedures was implemented. ${ }^{1}$ Coronary artery bypass grafting $(\mathrm{CABG})$ surgery is one high-risk procedure showing a consistent and comprehensive body of evidence, ${ }^{2-9}$ leading to a widespread belief that if all patients were treated at high-volume hospitals, thousands of lives would be saved each year. In the United States, in 2003, the Leapfrog hospital quality initiative established an annual hospital volume of $450 \mathrm{CABG}$ procedures to qualify for premium bonus payments over and above the established fee schedule. ${ }^{10}$

Currently, the literature on the $\mathrm{CABG}$ volume-outcome relationship is limited to inpatient or 30-day mortality, ${ }^{11}$ with little documentation on long-term outcomes. Using nationwide population-based data from Taiwan's National Health Insurance (NHI) database, we examined the association between hospitals' CABG surgery volumes and long-term (5-year) patient outcomes. Under NHI, all citizens ( $>96 \%$ of the island's 


\section{Abbreviations and Acronyms \\ $\mathrm{CABG}=$ coronary artery bypass grafting \\ ICD-9-CM = International Classification of Diseases- ninth revision-Clinical Modification \\ MACE = major adverse cardiovascular event \\ NHI $=$ National Health Insurance \\ PTCA = percutaneous transluminal coronary angioplasty}

population of about 23 million) have full choice of health care provider under a single health benefit plan, a comprehensive benefit package, and very low copayments. This structure enables virtually full access to all citizens when they perceive the need. Furthermore, all care, outpatient or inpatient, is captured in the claims database, permitting every long-term outcome to be tracked. This is unlike health systems, such as that of the United States, where patient segmentation by insurer, health plan, and provider panel disrupt the completeness of information on postdischarge events and outcomes. Furthermore, many other health care systems use gatekeeper or referral systems that limit provider choice, confounding volume-outcome studies. Taiwan's NHI claims data provide an opportunity to examine long-term outcomes without the above-mentioned sources of selection bias or follow-up bias.

In this study we use major adverse cardiovascular events (MACEs) as our outcome measure to examine the longterm effects of hospital volume. MACE outcomes include death, myocardial infarction, stroke, and repeat revascularization procedures (percutaneous transluminal coronary angioplasty [PTCA] or repeated CABG). Rather than limit to mortality alone, MACEs have been widely used to evaluate the outcomes of cardiac procedures in the current literature. ${ }^{12-14}$ To date, MACEs have been used to evaluate short-term outcomes of comparable/alternative care procedures. Our study is innovative in presenting evidence on long-term (rather than short-term) MACE outcomes (rather than mortality alone) over a 5-year follow-up period relative to hospital procedure volume.

\section{Materials and Methods Database}

We linked NHI claims data with Taiwan's Cause of Death file for the years 1997 through 2004. These data cover all inpatient and outpatient medical benefit claims for all citizens ( $>96 \%$ of the Taiwanese population [23 million]). Each claim has International Classification of Diseases-ninth revision-Clinical Modification (ICD-9-CM) codes for 1 principal operative procedure, 1 principal diagnosis, and up to 4 secondary diagnoses, along with details of the care provided, patient demographics, and provider characteristics.

The Cause of Death file provides data on the date of death and underlying cause of death (ICD-9-CM). Because of mandatory death registration, these data are accurate and comprehensive. Because these were deidentified secondary data released for public access for research purposes, the study was exempt from full review by the internal review board.

\section{Study Sample}

All in-patient claims for CABG surgery between January 1, 1997, and December 31, 1999, were screened for the following: (1) ICD-9 code 36.10-36.20 (bypass anastomosis for heart revascularization), (2) first-time CABG surgery for the patient, and (3) not being an emergency department admission. NHI was implemented in 1995, and paying for CABG operations out of pocket was beyond the reach of most Taiwanese before 1995. NHI claims since 1995 were scrutinized to verify that the sample cases were first-time CABG recipients. We excluded emergency CABG recipients (admitted through the emergency department) because these patients are documented to have disproportionately poor outcomes. ${ }^{15,16}$ Based on the above criteria, we generated a study sample of 5718 patients undergoing CABG surgery.

\section{Hospital CABG Volume Groups}

Based on unique hospital identifiers, we calculated each hospital's total $\mathrm{CABG}$ volume over the study period. Hospitals were then sorted, in ascending order, according to their total CABG volume, and cutoff points were determined by the volume that most closely sorted the sample patients into 4 quartile groups of roughly equivalent size. This is the standard documented method of classifying patients by the provider's procedure volume to obtain balanced cell sizes for the volume groups. ${ }^{6}$ The 4 hospital volume groups were 282 cases or less (low volume, $n=1584$ patients), 283 to 517 cases (medium volume, $\mathrm{n}=1317$ patients), 518 to 725 cases (high volume, $\mathrm{n}=1437$ patients), and 726 cases or more (very high volume, $\mathrm{n}=1380$ patients).

Study end points. The study objective was to examine postCABG MACE-free survival at 30 days and 5 years (the latter excluding patients with a MACE outcome within the first 30 days). This is because, relative to 5-year follow-up, 30-day MACE incidence is more likely driven by initial disease severity and by patient-specific clinical instability during the preoperative and postoperative phases.

We identified 4 clinical end points (MACE qualifying outcome) examined at 30 days and 5 years after each patient's operative date: death, myocardial infarction, stroke, and repeat revascularization procedure (PTCA or repeat CABG). During each patient's postCABG 30-day and 5-year periods, death (from Taiwan's Cause of Death file) or any subsequent inpatient claim or claims with a recording of a MACE event was logged into the study database. In addition, a composite MACE outcome was defined: any patient with 1 or more of the 4 events/outcomes above was defined as having a MACE outcome. The remaining patients were classified as MACE-free survivors at 30 days and 5 years (after 30 days). The composite definition of MACE-free survival in cardiovascular medicine has been widely used in the current literature. ${ }^{12-14}$

Variables of interest. The patient was the unit of analysis. The key independent variable was hospital volume. The dependent variables were dichotomous: having (or not having) a MACE within 30 days or 5 years (the denominator for the latter excluded patients with any MACEs within 30 days). Both outcomes were evaluated as hazard ratios by hospital volume.

We adjusted for patient and hospital characteristics. Patient variables included age, sex, income, and clinical severity at first 
admission, as well as CABG procedure attributes. Income was categorized as NT\$0 (not working), NT\$1 to NT\$15,840 (income less than minimum wage), NT\$15841 to NT\$25,000, and NT $\$ 25,001$ or greater. NT $\$ 15,840$ is Taiwan's minimum-wage level stipulated for a full-time employee. Clinical severity was captured by using 8 dichotomous (yes/no) variables: myocardial infarction (ICD 410), any other coronary artery disease (ICD 411-414), diabetes (ICD 250), chronic obstructive pulmonary disease (ICD 490-496), hypertension (ICD 401-405), renal dysfunction (ICD 580-586), congestive heart failure (ICD 4280), and stroke (ICD 430-438). We controlled for 2 CABG procedure attributes (partly representing unmeasured clinical severity and partly representing MACE propensity caused by operative complexity): concomitant valve repair (ICD procedure code 35 ) and use of an internal thoracic graft (ICD procedure code 36.15 and 36.16).

We adjusted for hospital and patient variables. Hospital variables included ownership (public, private not-for-profit [NFP], and for-profit [FP]), hospital level (medical center, $\geq 500$ beds; regional hospital, 250-499 beds; and district hospital, $\leq 20$ beds), and geographic location (north, south, east, and central Taiwan). Teaching status is excluded because all medical centers and regional hospitals are teaching hospitals, which would cause colinearity. Medical centers and regional hospitals, as well as notfor-profit status, are generally associated with state-of-the-art clinical technologic infrastructure. Medical centers and regional hospitals also have higher, round-the-clock, high-intensity monitoring of patients by residents, attending physicians, and teaching faculty, as well as nursing students of various levels. Therefore between the 2 variables, hospital size and ownership, the regression accounts for the facilities' clinical technology infrastructure and personnel support that might affect short-term and long-term outcomes.

\section{Statistical Analysis}

The SAS statistical package (Version 8.2; SAS Institute, Inc, Cary, NC) was used. Kaplan-Meier methods were used to estimate 30-day and 5-year (after 30 days) MACE-free survival, and their associations with hospital volume were assessed by using the log-rank test.

Survival time was computed from the surgical date to the date of MACE within the 30-day or (post-30-day) 5-year follow-up periods. Cox proportional hazard regressions yielded hazard ratios that estimate the contribution of hospital volume to MACE-free survival adjusted for hospital and patient characteristics.

\section{Results}

Table 1 describes the distribution of the sample patients by using severity measures at the time of CABG and CABG procedure attributes. Of 5718 first-time CABG hospitalizations, the majority $(76.8 \%)$ of patients was male, the mean age was 69.4 years (SD, 9.9), 99.2\% had either myocardial infarction or other coronary artery disease, and $29.1 \%, 38.4 \%$, and $6.5 \%$ had diabetes, hypertension, and stroke, respectively.

Hospital and patient characteristics by hospital CABG volume groups are presented in Table 2. Mean hospital CABG volume for the study period was 214 operations. The majority of low-volume hospitals were regional and
TABLE 1. Distribution of first-time CABG patients in Taiwan, 1997-1999

\begin{tabular}{|c|c|c|}
\hline \multirow[b]{2}{*}{ Variables } & \multicolumn{2}{|c|}{ Totals* } \\
\hline & No. & $\%$ \\
\hline \multicolumn{3}{|l|}{ Hospital volume group } \\
\hline Low volume & 1584 & 27.7 \\
\hline Medium volume & 1317 & 23.0 \\
\hline High volume & 1437 & 25.1 \\
\hline Very high volume & 1380 & 24.1 \\
\hline \multicolumn{3}{|l|}{ Patient sex } \\
\hline Male & 4389 & 76.8 \\
\hline Female & 1329 & 23.2 \\
\hline \multicolumn{3}{|l|}{ Patient age (y) } \\
\hline$<65$ & 1534 & 26.8 \\
\hline $65-74$ & 2278 & 39.8 \\
\hline$>74$ & 1906 & 33.3 \\
\hline \multicolumn{3}{|l|}{ Coronary disease } \\
\hline $\begin{array}{c}\text { MI as primary diagnosis or } \\
\text { secondary diagnosis }\end{array}$ & 92 & 1.6 \\
\hline Other coronary artery disease & 5582 & 97.6 \\
\hline No coronary artery disease & 44 & 0.8 \\
\hline \multicolumn{3}{|l|}{ Diabetes } \\
\hline Yes & 1662 & 29.1 \\
\hline No & 4056 & 70.9 \\
\hline \multicolumn{3}{|l|}{ Hypertension } \\
\hline Yes & 2196 & 38.4 \\
\hline No & 3522 & 61.6 \\
\hline \multicolumn{3}{|l|}{ COPD } \\
\hline Yes & 228 & 4.0 \\
\hline No & 5490 & 96.0 \\
\hline \multicolumn{3}{|l|}{ Renal disease } \\
\hline Yes & 416 & 7.3 \\
\hline No & 5302 & 92.7 \\
\hline \multicolumn{3}{|l|}{ Congestive heart failure } \\
\hline Yes & 411 & 7.2 \\
\hline No & 5307 & 92.8 \\
\hline \multicolumn{3}{|l|}{ Stroke } \\
\hline Yes & 371 & 6.5 \\
\hline No & 5347 & 93.5 \\
\hline \multicolumn{3}{|l|}{ Concomitant valve repair } \\
\hline Yes & 401 & 7.0 \\
\hline No & 5317 & 93.0 \\
\hline \multicolumn{3}{|l|}{ Internal thoracic artery graft } \\
\hline Yes & 1141 & 20.0 \\
\hline No & 4577 & 80.0 \\
\hline
\end{tabular}

$M I$, Myocardial infarction; COPD, chronic obstructive pulmonary disease. ${ }^{*}$ Total patient sample $=5718$.

not-for-profit hospitals, and all very high-volume hospitals were teaching hospitals (medical centers or regional hospitals).

The in-hospital mortality rates were $3.5 \%, 3.9 \%, 2.9 \%$, and $3.1 \%$ for low-, medium-, high- and very high-volume hospital groups, respectively. The 30-day mortality rates were $4.2 \%$, $4.3 \%, 4.2 \%$, and $3.2 \%$ for low-, medium-, high- and very 
TABLE 2. Hospital and patient characteristics in Taiwan by hospital CABG volume groups, 1997-1999

\begin{tabular}{|c|c|c|c|c|c|c|c|c|c|c|c|c|}
\hline \multirow[b]{3}{*}{ Variable } & \multicolumn{12}{|c|}{ Hospital CABG volume group } \\
\hline & \multicolumn{3}{|c|}{ Low ( $\leq 282)$} & \multicolumn{3}{|c|}{ Medium (283-517) } & \multicolumn{3}{|c|}{ High (518-725) } & \multicolumn{3}{|c|}{ Very high $(\geq 726)$} \\
\hline & No. & $\%$ & Mean (SD) & No. & $\%$ & Mean (SD) & No. & $\%$ & Mean (SD) & No. & $\%$ & Mean (SD) \\
\hline \multicolumn{13}{|l|}{ Hospital characteristics* } \\
\hline No. of Hospitals & 32 & & & 8 & & & 4 & & & 3 & & \\
\hline Mean of hospital CABG volume & & & $95(87)$ & & & $445(66)$ & & & $698(28)$ & & & $922(136)$ \\
\hline \multicolumn{13}{|l|}{ Hospital level } \\
\hline Medical center & 7 & 21.9 & & 6 & 75.0 & & 2 & 50.0 & & 2 & 66.7 & \\
\hline Regional hospital & 21 & 65.6 & & 2 & 25.0 & & 1 & 25.0 & & 1 & 33.3 & \\
\hline District hospital & 4 & 12.5 & & - & - & & 1 & 25.0 & & - & & \\
\hline \multicolumn{13}{|l|}{ Hospital ownership } \\
\hline Public & 7 & 21.9 & & 2 & 25.0 & & 2 & 50.0 & & 2 & 66.7 & \\
\hline Private (not-for-profit) & 16 & 50.0 & & 5 & 62.5 & & 2 & 50.0 & & 1 & 33.3 & \\
\hline Private (for-profit) & 9 & 28.1 & & 1 & 12.5 & & - & - & & - & - & \\
\hline \multicolumn{13}{|l|}{ Hospital Location } \\
\hline Northern & 11 & 34.4 & & 3 & 37.5 & & 2 & 50.0 & & 3 & 100 & \\
\hline Central & 11 & 34.4 & & 1 & 12.5 & & 1 & 25.0 & & - & - & \\
\hline Southern & 9 & 28.1 & & 4 & 50.0 & & 1 & 25.0 & & - & - & \\
\hline Eastern & 1 & 3.1 & & - & - & & - & - & & - & - & \\
\hline \multicolumn{13}{|l|}{ Patient characteristics $\dagger$} \\
\hline Total no. of Patients & 1584 & 27.7 & & 1317 & 23.0 & & 1437 & 25.1 & & 1380 & 24.1 & \\
\hline Mean age of patients (y) & & & $68.8(9.8)$ & & & $69.3(9.7)$ & & & $68.4(10.4)$ & & & $71.0(9.3)$ \\
\hline \multicolumn{13}{|l|}{ Patient sex } \\
\hline Male & 1155 & 72.9 & & 1006 & 76.4 & & 1086 & 75.6 & & 1142 & 82.8 & \\
\hline Female & 429 & 27.1 & & 311 & 23.6 & & 351 & 24.4 & & 238 & 17.3 & \\
\hline \multicolumn{13}{|l|}{ Patient age } \\
\hline$<65 \mathrm{y}$ & 453 & 28.6 & & 349 & 26.5 & & 445 & 31.0 & & 287 & 20.8 & \\
\hline $65-74$ y & 643 & 40.6 & & 547 & 41.5 & & 582 & 40.5 & & 506 & 36.7 & \\
\hline$>74 y$ & 488 & 30.8 & & 421 & 32.0 & & 410 & 28.5 & & 587 & 42.5 & \\
\hline \multicolumn{13}{|l|}{ Coronary disease } \\
\hline $\begin{array}{l}\text { MI as primary or } \\
\text { secondary diagnosis }\end{array}$ & 39 & 2.5 & & 12 & 0.9 & & 14 & 1.0 & & 27 & 2.0 & \\
\hline $\begin{array}{l}\text { Other coronary } \\
\text { artery disease }\end{array}$ & 1530 & 96.5 & & 1301 & 98.8 & & 1411 & 98.2 & & 1340 & 97.1 & \\
\hline $\begin{array}{l}\text { No coronary } \\
\text { artery disease }\end{array}$ & 15 & 1.0 & & 4 & 0.3 & & 12 & 0.8 & & 13 & 0.9 & \\
\hline \multicolumn{13}{|l|}{ Diabetes } \\
\hline Yes & 540 & 34.1 & & 318 & 24.2 & & 364 & 25.3 & & 440 & 31.9 & \\
\hline No & 1044 & 65.9 & & 999 & 75.8 & & 1073 & 74.7 & & 940 & 68.1 & \\
\hline \multicolumn{13}{|l|}{ Hypertension } \\
\hline Yes & 645 & 40.7 & & 391 & 29.7 & & 548 & 38.1 & & 612 & 44.4 & \\
\hline No & 939 & 59.3 & & 926 & 70.3 & & 869 & 61.9 & & 768 & 55.6 & \\
\hline \multicolumn{13}{|l|}{ COPD } \\
\hline Yes & 64 & 4.0 & & 25 & 1.9 & & 61 & 4.2 & & 78 & 5.7 & \\
\hline No & 1520 & 96.0 & & 1292 & 98.1 & & 1376 & 95.8 & & 1302 & 94.3 & \\
\hline Renal disease & & & & & & & & & & & & \\
\hline Yes & 145 & 9.2 & & 82 & 6.2 & & 88 & 6.1 & & 101 & 7.3 & \\
\hline No & 1439 & 90.8 & & 1235 & 93.8 & & 1349 & 93.9 & & 1279 & 92.7 & \\
\hline Congestive heart failure & & & & & & & & & & & & \\
\hline Yes & 167 & 10.5 & & 38 & 2.9 & & 152 & 10.6 & & 54 & 3.9 & \\
\hline No & 1417 & 89.5 & & 1279 & 97.1 & & 1285 & 89.4 & & 1326 & 96.1 & \\
\hline Stroke & & & & & & & & & & & & \\
\hline Yes & 120 & 7.6 & & 58 & 4.4 & & 74 & 5.2 & & 119 & 8.6 & \\
\hline No & 1464 & 92.4 & & 1259 & 95.6 & & 1363 & 94.8 & & 1261 & 91.4 & \\
\hline
\end{tabular}




\begin{tabular}{|c|c|c|c|c|c|c|c|c|c|c|c|c|}
\hline \multirow[b]{3}{*}{ Variable } & \multicolumn{12}{|c|}{ Hospital CABG volume group } \\
\hline & \multicolumn{3}{|c|}{ Low ( $\leq 282)$} & \multicolumn{3}{|c|}{ Medium (283-517) } & \multicolumn{3}{|c|}{ High (518-725) } & \multicolumn{3}{|c|}{ Very high $(\geq 726)$} \\
\hline & No. & $\%$ & Mean (SD) & No. & $\%$ & Mean (SD) & No. & $\%$ & Mean (SD) & No. & $\%$ & Mean (SD) \\
\hline \multicolumn{13}{|c|}{ Concomitant valve repair } \\
\hline Yes & 107 & 6.8 & & 78 & 5.9 & & 100 & 7.0 & & 116 & 8.4 & \\
\hline No & 1477 & 93.2 & & 1239 & 94.1 & & 1337 & 93.0 & & 1264 & 91.6 & \\
\hline \multicolumn{13}{|l|}{$\begin{array}{c}\text { Internal thoracic } \\
\text { artery graft }\end{array}$} \\
\hline Yes & 134 & 8.5 & & 260 & 19.7 & & 514 & 35.8 & & 233 & 16.9 & \\
\hline No & 1450 & 91.5 & & 1057 & 80.3 & & 923 & 64.2 & & 1147 & 83.1 & \\
\hline \multicolumn{13}{|c|}{$\begin{array}{l}\text { Income-related insured } \\
\text { amount }\end{array}$} \\
\hline 0 & 557 & 35.2 & & 432 & 32.8 & & 552 & 38.4 & & 350 & 25.4 & \\
\hline NT\$1 15,840 & 400 & 25.3 & & 478 & 36.3 & & 275 & 19.1 & & 719 & 52.1 & \\
\hline NT\$15,841 25,000 & 498 & 31.4 & & 296 & 22.5 & & 468 & 32.6 & & 208 & 15.1 & \\
\hline$\geq \mathrm{NT} \$ 25,001$ & 129 & 8.1 & & 111 & 8.4 & & 142 & 9.9 & & 103 & 7.5 & \\
\hline
\end{tabular}

$C A B G$, Coronary artery bypass grafting; $S D$, standard deviation; $M I$, myocardial infarction; COPD, chronic obstructive pulmonary disease. ${ }^{*}$ Total number of hospitals $=47 . \dagger$ Total patient sample $=5718$.

high-volume hospital groups, respectively (data not shown). Examined on the basis of individual MACE type, hospital CABG volume is inversely associated with the 4 end points and the composite end point, MACE, as shown in Table 3. During the 5 years after CABG, low-volume hospitals had the highest stroke and repeat CABG/PTCA rates, which systematically decreased with increasing volume category, although the differences did not attain statistical significance. However, mortality accounted for a small proportion $(9 \%$ ) of all MACE events (range, $6 \%$ to $12 \%$ across volume groups). When the cause of mortality was investigated, about one third of deaths were due to cardiovascular causes, with the distribution of cardiovascular and noncardiovascular causes being similar across the 4 volume groups (data not shown).

The crude 30-day and 5-year MACE-free survival rates, as well as adjusted hazard ratios by hospital volume groups, are provided in Table 4, showing increasing 30-day and 5-year MACE-free survival rates with increasing hospital volume.
Crude hazard ratio for a 30-day and 5-year MACE event decreased with increasing hospital volume (30-day crude hazard ratio for low-volume hospitals of 1.585 [reciprocal of 0.631 ] relative to very high-volume hospitals). After adjusting for patient demographics, initial case severity, $\mathrm{CABG}$ procedure attributes, and hospital characteristics, the negative association between hospital volume and 30-day and 5-year MACE hazard is sustained. Increasing hospital volume predicts a systematic decrease in adjusted MACE hazard at 5 years. The 30-day MACE hazard ratios for patients in low-volume hospitals was $1.502(P<.05)$ relative to very high-volume hospitals. The 5-year MACE hazard ratios for patients in low-volume hospitals were $1.131(P<.01)$ and $1.233(P<.001)$ relative to those of patients in high-volume and very high-volume hospitals.

\section{Discussion}

This study makes a new contribution to the CABG volumeoutcome literature by investigating MACE hazard rather

TABLE 3. Five-year follow-up results (excluding those with a MACE within 30 days) by hospital CABG volumes, 1997-1999

\begin{tabular}{|c|c|c|c|c|c|c|c|c|c|c|}
\hline & \multicolumn{2}{|c|}{ Mortality } & \multicolumn{2}{|c|}{ Myocardial infarction } & \multicolumn{2}{|c|}{ Stroke } & \multicolumn{2}{|c|}{ Repeat CABG or PTCA } & \multicolumn{2}{|c|}{ All MACE } \\
\hline & Yes & No & Yes & No & Yes & No & Yes & No & Yes & No \\
\hline Variables & No. $(\%)$ & No. $(\%)$ & No. $(\%)$ & No. $(\%)$ & No. $(\%)$ & No. $(\%)$ & No. $(\%)$ & No. $(\%)$ & No. $(\%)$ & No. $(\%)$ \\
\hline \multicolumn{11}{|c|}{ Hospital CABG volume } \\
\hline$\leq 282$ & $50(3.3)$ & $1456(96.7)$ & $28(1.9)$ & $1478(98.1)$ & $165(11.0)$ & $1341(89.0)$ & $179(11.9)$ & $1327(88.1)$ & $422(28.0)$ & $1084(72.0)$ \\
\hline $283-517$ & $20(1.6)$ & $1235(98.4)$ & $13(1.0)$ & $1242(99.0)$ & $131(10.4)$ & $1124(89.6)$ & $143(11.4)$ & 1112 (88.6) & $307(24.5)$ & $948(75.5)$ \\
\hline $518-725$ & $25(1.8)$ & $1347(98.2)$ & $20(1.5)$ & $1352(98.5)$ & $134(9.8)$ & $1238(90.2)$ & $138(10.0)$ & $1234(90.0)$ & $317(23.1)$ & $1055(76.9)$ \\
\hline$\geq 726$ & $26(1.9)$ & $1308(98.1)$ & $14(1.0)$ & $1320(99.0)$ & $129(9.7)$ & $1205(90.3)$ & $106(7.9)$ & $1228(92.1)$ & $275(20.6)$ & $1059(79.4)$ \\
\hline
\end{tabular}

Total patient sample $=5467 . M A C E$, Major adverse cardiovascular event; $C A B G$, coronary artery bypass grafting; PTCA, percutaneous transluminal coronary angioplasty. 
TABLE 4. Thirty-day and 5-year MACE-free survival (excluding those with a MACE within $\mathbf{3 0}$ days) and hazard ratios by hospital CABG volume groups

\begin{tabular}{|c|c|c|c|}
\hline Variables & $\begin{array}{l}\text { 30-d MACE event-free } \\
\text { survival rate (\%) }\end{array}$ & $\begin{array}{l}\text { Crude hazard } \\
\text { ratio }(95 \% \mathrm{CI})\end{array}$ & $\begin{array}{l}\text { Adjusted hazard } \\
\text { ratio* }(95 \% \mathrm{CI})\end{array}$ \\
\hline \multicolumn{4}{|c|}{ Hospital CABG volume $\dagger$} \\
\hline$\leq 282$ & 95.1 & 1.000 & 1.000 \\
\hline $283-517$ & 95.3 & $1.019(0.708-1.465)$ & $0.952(0.671-1.349)$ \\
\hline $518-725$ & 95.5 & $0.780(0.547-1.113)$ & $0.922(0.659-1.288)$ \\
\hline \multirow[t]{2}{*}{$\geq 726$} & 96.7 & $0.631(0.428-0.932) \ddagger$ & $0.666(0.459-0.967) \ddagger$ \\
\hline & $\begin{array}{c}\text { 5-year MACE event-free } \\
\text { survival }(\%)\end{array}$ & $\begin{array}{l}\text { Crude hazard } \\
\text { ratio }(95 \% \mathrm{CI})\end{array}$ & $\begin{array}{l}\text { Adjusted hazard } \\
\text { ratio* }(95 \% \mathrm{CI})\end{array}$ \\
\hline \multicolumn{4}{|c|}{ Hospital CABG volume } \\
\hline$\leq 282$ & 72.0 & 1.000 & 1.000 \\
\hline $283-517$ & 75.5 & $0.882(0.797-0.976) \ddagger$ & $0.912(0.812-1.081)$ \\
\hline $518-725$ & 76.9 & $0.855(0.769-0.949) \S$ & $0.884(0.809-0.965) \S$ \\
\hline$\geq 726$ & 79.4 & $0.765(0.689-0.850) \|$ & $0.811(0.728-0.904) \|$ \\
\hline
\end{tabular}

MACE, Major adverse cardiovascular event; $C A B G$, coronary artery bypass grafting; $C l$, confidence interval. *Hazard ratios are adjusted for the patient's age, sex, income-linked premium category, comorbidities, procedure attributes, and hospital characteristics, including hospital ownership, hospital level, and geographic location. $\dagger$ Total sample size $=5718 . \ddagger P<.05, \S P<.01$, and $\| P<.001$. .Total sample size $=5467$.

than mortality alone and by examining long-term MACEfree survival rather than 30-day or inpatient mortality, which has been the norm in these types of studies. It also distinguishes between 30-day and 5-year MACEs (after 30-days) to sequester the high and variable vulnerability of the first 30 days from the relatively nonvolatile, post-30-day period. Other key features that qualify its unique contribution are the following: (1) the data's ability to capture every subsequent MACE event treated anywhere in Taiwan and linkages to the Cause of Death data file and (2) coverage of all CABG age groups throughout the country, unlike most documented literature from the United States that focuses exclusively on the Medicare population because of the inability to followup younger age groups because of periodic churning in insurance status and plan type. Although biases from these several sources have confounded past efforts to investigate the true relationship of provider volume with long-term outcomes, our study is able to show that unequivocally, hospital CABG volume affects MACE outcomes. Although our study might not be readily replicable in many countries because of a lack of such a data source, it makes a signal contribution by providing empiric evidence for policymakers to benefit the populace and to reduce health care costs arising from careintensive MACE outcomes.

Our findings indicate that the favorable association between provider volume and in-hospital/30-day mortality $^{5-10}$ extends beyond the immediate postoperative period to 5 years after the operation. Furthermore, our study also indicates that not only mortality but also intermediate events and repeat revascularization in the following 5 years are less frequent among patients treated by higher-volume providers after adjusting for patient severity and other clinical charac- teristics, demographics, and income, as well as hospital characteristics.

Our study also confirms that mortality represents the tip of the iceberg of adverse outcomes (only 9\% of all MACEs). It demonstrates that limiting $\mathrm{CABG}$ outcome studies to mortality alone would greatly underestimate the effect of volume or, worse, fail to detect long-term outcome differences across volume groups. This is one likely reason why the only documented study of long-term mortality versus provider volume ${ }^{11}$ contradicts our finding. They reported no difference in risk-adjusted 3-year survival at a low-volume hospital compared with a high-volume hospital, when the same high-volume surgeons performed the procedure. Other reasons for their finding could be the following. First, they compared 1 low-volume hospital in Ohio with 1 highvolume hospital for a 5-surgeon team. Therefore lack of statistical power to compare hospital groups would preclude statistically relevant conclusions. Second, in the United States high-volume hospitals in metropolitan areas are very likely teaching hospitals that are also safety net providers to treat the uninsured, Medicaid, and African American populations. Their patient profile confirms the significantly higher proportions for these groups among the high-volume hospital's patients. Poorer risk-adjusted outcomes of CABG operations (as well as most high-risk and chronic conditions) among these groups is well documented. Therefore their finding of no difference between the low-volume and high-volume hospital could actually represent better average performance by the high-volume hospital when the generally poor risk-adjusted outcomes of the African American, uninsured, and Medicaid population is taken into account. 
To compare our study with theirs, we evaluated mortality alone, and yet we find that high volume predicts lower adjusted mortality hazard. Because Taiwan has a universal coverage, single-payer system with low copayments and an ethnically homogeneous population, differences in treatment by insurance status is not a source of confounding, rendering our finding robust to extraneous confounding.

To explain our finding, 2 hypotheses are documented in the literature. One is that "practice makes perfect," causing high-volume providers to have in place better care procedures, recognition of potential complications before they supervene, and better care organizations right up to discharge. ${ }^{17}$ The second documented hypothesis is that "self-referral" might cause more patients to attend hospitals with a reputation for better outcomes, causing such hospitals to become high-volume hospitals. ${ }^{18}$ Although the former is possible, the latter might also play a role in Taiwan ${ }^{19,20}$ because of full provider choice, a considerable density of hospitals of all types in the populated areas, and good transportation systems.

Additionally, some researchers have suggested that the volume-outcome relationship in CABG surgery might result from systematic differences in patient severity between low- and high-volume providers. ${ }^{21,22}$ Many opponents of regionalization policies have argued that administrative databases might not permit adequate risk adjustment. We submit that our adjustment for a very comprehensive array of clinical risk factors and the $\mathrm{CABG}$ attributes of internal thoracic artery graft use and concomitant valve repair should have accounted for most of the patient-specific vulnerabilities to MACEs. Moreover, we excluded all CABG cases admitted through the emergency department, which also precludes a major source of variance in clinical severity. Furthermore, it must be noted that, in general, severely ill but not emergency patients are most likely to choose medical centers or regional hospitals (which are also large and noted for their state-of-the-art technologic infrastructure). Therefore it is unlikely that systematically higher unmeasured severity at low-volume hospitals is driving our findings. Consistent with this reasoning, patients treated at very high-volume hospitals had higher rates of concomitant valve repair and the use of internal thoracic artery grafts than other volume groups. Other studies have also shown that low-risk patients for cardiac interventions were more likely to be treated by low-volume providers. ${ }^{23}$ Another question could be that patients of lower socioeconomic status could be disproportionately represented among the low-volume hospitals' patient panels. Our adjustment for patient's income-linked premium deduction category takes care of this potential source of confounding.

Finally, it could be argued that 5-year MACE differences between low- and high-volume hospitals could be due to lower all-cause mortality among the high-volume hospital patients, in turn because of generally better medical and surgical inpatient care for noncardiovascular morbidities subsequent to their CABG surgery. Our finding that the distribution of cardiovascular versus other cause of death is similar across the volume groups rebuts this potential explanation for our findings. Moreover, as explained earlier, mortality contributes to less than $10 \%$ of total MACE incidence; the sentinel cardiovascular MACEs independently show a systematic (adjusted) decrease with increasing hospital volume.

There are some study limitations. First, the claims database lacks information on the clinical history of the patient, such as cigarette smoking, alcohol consumption, left ventricular ejection fraction, level of block and number of arteries blocked, body mass index, creatinine levels, and clinical severity scores. Yet with the large sample used, it is unlikely that systematic differences in these factors are driving our findings. A second limitation is that because of overall lower CABG volumes in Taiwan compared with the United States or other developed countries, we were unable to use the conventional hospital volume thresholds documented in the literature for comparability of our findings across countries.

Notwithstanding the above limitations, the findings suggest that high-volume hospitals have some processes, infrastructure/personnel factors, or both that seem to produce not only better short-term outcomes but also better longterm outcomes. All high-volume hospitals are teaching hospitals. Detailed comparative studies are necessary of the care processes, medical and nursing monitoring, technicalsupport infrastructure, and care provider interactions with patients to understand what makes for better long-term outcomes.

Theoretically, short-term outcomes could be attributed to better surgical skills because of higher surgeon volumes. Yet analysis of the above data by surgeon volume instead of hospital volume does not show an association (data not shown). Our finding that hospital volume, but not surgeon volume, predicts adverse long-term outcomes is plausible for a high-risk procedure such as CABG performed on highrisk patients. The speedy and appropriate response of a hospital's preoperative and postoperative technical support and skilled medical/nursing manpower can make or break a patient's MACE vulnerability.

Intuitively, one would expect that the above attributes would affect short-term MACE survival, but one might question why these institutional attributes would affect long-term outcome. One possibility is that the high intensity of qualified manpower in a teaching institution (eg, residents, medical students, nursing students, and dieticians) might result in a greater probability of appropriate advice and health education support to patients to attend regular and full-scale checkup visits, as well as to change their lifestyle and risk factors after leaving the hospital.

Although the above explanations are clearly speculative, they are presented as possible mediating variables underlying our finding. The differences in long-term outcomes in 
addition to short-term outcomes amounts to $50 \%$ greater odds of a MACE within 30 days (reciprocal of 0.666) among patients in low-volume hospitals (adjusted for severity, comorbidities, patient demographics, and other factors) and an additional 23\% greater (adjusted) MACE odds after 30 days but within 5 years (reciprocal of 0.811). This magnitude of difference alone justifies detailed process studies by peer task forces to leverage our findings into suitable professional and policymaker interventions. The answer might not necessarily be regionalization of high-risk procedures because some low-volume hospitals can produce excellent longterm outcomes and some high-volume hospitals can provide poor outcomes. Only a detailed study of care processes and services common to high-volume hospitals can provide guidance to address this issue.

\section{References}

1. Nallamothu BK, Eagle KA, Ferraris VA, Sade RM. Should coronary artery bypass grafting be regionalized? Ann Thorac Surg. 2005;80: $1572-81$.

2. Wu C, Hannan EL, Ryan TJ, Bennett E, Culliford AT, Gold JP, et al. Is the impact of hospital and surgeon volumes on the in-hospital mortality rate for coronary artery bypass graft surgery limited to patients at high risk? Circulation. 2004;110:784-9.

3. Hannan EL, Kilburn H, Bernard H, O'Donnell JF, Lukacik G, Shields EP. Coronary artery bypass surgery: the relationship between in-hospital mortality rate and surgical volume after controlling for clinical risk factors. Med Care. 1991;29:1094-107.

4. Hannan EL, O’Donnell JF, Kilburn H, Bernard HR, Yazici A. Investigation of the relationship between volume and mortality for surgical procedures performed in New York State hospitals. JAMA. 1989;262: 503-10.

5. Rathore SS, Epstein AJ, Volpp KG, Krumholz HM. Hospital coronary artery bypass graft surgery volume and patient mortality, 1998-2000. Ann Surg. 2004;39:110-7.

6. Birkmeyer JD, Siewers AE, Finlayson EV, Stukel TA, Lucas FL, Batista I, et al. Hospital volume and surgical mortality in the United States. N Engl J Med. 2002;346:1128-37.

7. Hannan EL, Wu C, Ryan TJ, Bennett E, Culliford AT, Gold JP, et al. Do hospitals and surgeons with higher coronary artery bypass graft surgery volumes still have lower risk-adjusted mortality rates? Circulation. 2003;108:795-801.

8. Hannan EL, Siu AL, Kumar D, Kilburn H, Chassin MR. The decline in coronary artery bypass graft surgery mortality in New York State. The role of surgeon volume. JAMA. 1995;273:209-13.

9. Showstack JA, Rosenfeld KE, Garnick DW, Luft HS, Schaffarzick RW, Fowles J. Association of volume with outcome of coronary artery bypass graft surgery. Scheduled vs nonscheduled operations. JAMA. 1987;257: 785-9.

10. Shahian DM. Improving cardiac surgery quality-volume, outcome, process? JAMA. 2004;291:246-8.

11. Zacharias A, Schwann TA, Riordan CJ, Durham SJ, Shah A, Papadimos TJ, et al. Is hospital procedure volume a reliable marker of quality for coronary artery bypass surgery? A comparison of risk and propensity adjusted operative and midterm outcomes. Ann Thorac Surg. 2005;79:1961-9.

12. Youssef AA, Chang LT, Hang CL, Wu CJ, Cheng CI, Yang CH, et al. Level and value of interleukin-18 in patients with acute myocardial infarction undergoing primary coronary angioplasty. Circ J. 2007;71: 703-8.

13. Wang TY, Hasselblad V, Peterson JL, Wijns W, Parhizgar A, Kereiakes DJ, et al. The Cobalt chromium STent with Antiproliferative for Restenosis II (COSTAR II) trial study design: advancing the activecontrol evaluation of second-generation drug-eluting stents. Am Heart J. 2007; 153:743-8.

14. Rodriguez AE, Baldi J, Fernández Pereira C, Navia J, Rodriguez Alemparte M, et al. Five-year follow-up of the Argentine randomized trial of coronary angioplasty with stenting versus coronary bypass surgery in patients with multiple vessel disease (ERACI II). J Am Coll Cardiol. 2005;46:582-8.

15. Tomasco B, Cappiello A, Fiorilli R, Leccese A, Lupino R, Romiti A, et al. Surgical revascularization for acute coronary insufficiency: analysis of risk factors for hospital mortality. Ann Thorac Surg. 1997;64:678-83.

16. Chen Y, Almeida AA, Goldstein J, Shardey GC, Pick AW, Moshinsky R, et al. Urgent and emergency coronary artery bypass grafting for acute coronary syndromes. ANZ J Surg. 2006;76:769-73.

17. Luft HS, Bunker JP, Enthoven AC. Should operations be regionalized? The empirical relation between surgical volume and mortality. $N$ Engl J Med. 1979;301:1364-9.

18. Jollis JG, Peterson ED, Nelson CL, Stafford JA, DeLong ER, Muhlbaier LH, et al. Relationship between surgeon and hospital coronary angioplasty volume and outcome in elderly patients. Circulation. 1997; $95: 2485-91$

19. Cheng SH, Song HY. Surgeon performance information and consumer choice: a survey of subjects with the freedom to choose between doctors. Qual Saf Health Care. 2004;13:98-101.

20. Wen HC, Tang CH, Lin HC, Tsai CS, Chen CS, Li CY. Association between surgeon and hospital volume in coronary artery bypass graft surgery outcomes: a population-based study. Ann Thorac Surg. 2006; 81:835-42.

21. Shahian DM, Normand SL. The volume-outcome relationship: from Luft to Leapfrog. Ann Thorac Surg. 2003;75:1048-58.

22. Halm EA, Lee C, Chassin MR. Is volume related to outcome in health care? A systematic review and methodologic critique of the literature. Ann Intern Med. 2002;137:511-20.

23. Shook TL, Sun GW, Burstein S, Eisenhauer AC, Matthews RV. Comparison of percutaneous transluminal coronary angioplasty outcome and hospital costs for low-volume and high-volume operators. Am J Cardiol. 1996;77:331-6. 